\title{
Adherence to Multiple Medications Prescribed for a Chronic Disease: A Methodological Investigation
}

\author{
Ramsankar Basak, PhD; David J. McCaffrey, III, RPh, PhD; John P. Bentley, PhD; \\ Sarahmona M. Przybyla, PhD; Donna West-Strum, RPh, PhD; and Benjamin F. Banahan, III, PhD
}

\begin{abstract}
BACKGROUND: Many patients receive multiple medications for the treatment of a disease. While monitoring adherence is important, a composite measure of adherence is useful for estimating adherence to multiple medications in these patients. There are multiple ways to compute composite estimates of adherence to multiple medications, including (a) $80 \%$ of days covered by at least 1 medication ("at least 1"); (b) $80 \%$ of days covered by both medications ("both"); (c) $80 \%$ of days covered by each medication measured separately ("all"); and (d) computing an average of the individual medication adherence estimates ("average"). Comparison of adherence rates to individual medications and that of composite estimates are important for intervention decisions and effective disease management.
\end{abstract}

OBJECTIVES: To (a) examine adherence to multiple medications prescribed for a disease; (b) estimate composite adherence to multiple medications prescribed for a disease; and (c) determine the rate of differential classification of a patient being adherent as is estimated by different available algorithms.

METHODS: A retrospective cohort study was designed using 2002-2003 MarketScan Commercial Claims and Encounters data. To be included in the cohort, patients had to be less than aged 65 years and had to have separate prescriptions filled for 2 classes of diabetes medications (i.e., any sulfonylurea [SU] and any thiazolidinedione [TZD]) at least once; patients taking other diabetic medications over the observation period were excluded. Adherence was measured by proportion of days covered (PDC) over periods of 90 days ( 8 quarters total) and cumulatively over the 2-year study period. For some composite adherence estimates, patients were considered adherent if $P D C \geq 80 \%$. Survival curves using the life-table method were constructed to compare the time until PDC became $<80 \%$ as estimated by the 3 different categorical composite measures.

RESULTS: A total of 6,043 patients were included in the analysis. Across the 8 quarters under consideration, the average PDC estimates ranged between $69.8 \%-84.2 \%$ for SUs and $70.3 \%-85.6 \%$ for TZDs. The mean composite PDC based on the average algorithm varied between $69.4 \%$ and $84.9 \%$ when measured over each quarter or cumulatively. Similarly, the rates of composite adherence ranged from $74.5 \%$ to $88.2 \%, 46.4 \%$ to $61.2 \%$, and $47.7 \%$ to $62.9 \%$ for the "at least 1," "both," and "all" methods, respectively. Many subjects were classified as adherent by 1 composite dichotomous measure but not by all 3 dichotomous measures (i.e., "all," "at least 1," and "both"); of these patients, $30.6 \%-38.2 \%$ were classified differently as to their adherence status over different quarters by different measures. Survival curves of categorical composite measures were different $(P<0.05)$ from one another. "At least 1 " identified more patients as persistent and showed a much slower decline than did the "all" or "both" approaches.

CONCLUSIONS: Subjects were found to have a level of adherence-as estimated by individual medication adherence and composite adherence metrics-for multiple medications prescribed for a disease that, while not optimal from the perspective of patient care, was not entirely poor. In addi- tion, composite estimates of adherence considerably varied depending on algorithms used. Most importantly, a large number of patients appeared to be subject to inconsistent classification based on adherence measurement algorithm. Adherence estimates produced by different composite measurement approaches give rise to difficulty in consistent interpretation, which may be detrimental to appropriate patient care decision making.

J Manag Care Pharm. 2014;20(8):815-23

Copyright $\odot 2014$, Academy of Managed Care Pharmacy. All rights reserved.

\section{What is already known about this subject}

The trend of prescribing multiple medications for the treatment of a disease has been increasing.

There are several ways to compute a composite measure of adherence to multiple medications prescribed for a disease.

Depending on algorithms of a composite measure of multiple medication adherence, variability in overall adherence estimates occurs.

\section{What this study adds}

In diabetes patients, adherence to 1 diabetes medication follows that of another diabetes medication over a period of observation. Whether or not a patient will be classified as adherent to multiple medications may depend on the algorithm used to compute a composite measure of adherence to multiple medications.

Depending on the use of algorithms of a composite measure of multiple medication adherence, a large proportion of patients are likely to be inconsistently classified as adherent.

Different composite measurement algorithms result in statistically different population persistence estimates.

T he practice of using multiple medications occurs in many chronic diseases, such as diabetes or hypertension, and in treating underlying causal factors of a disease (e.g., heart failure). ${ }^{1,2}$ While adherence to medication is associated with positive health outcomes, in general, rates of adherence to or long-term persistence with chronic medication regimens are poor. ${ }^{3-5}$ Thus, monitoring and measuring adherence behavior is critical to chronic disease management. As is evidenced by the published literature, there are several different methods that can be used to measure medication adherence (e.g., pill 
counts and self-report). ${ }^{6}$ Among the approaches available to researchers is the use of administrative claims data. The availability of and use of prescription claims data has resulted in the use of different approaches to the measurement of adherence to multiple medications that are concurrently consumed for a single disease. For example, Choudhry et al. (2009) presented multiple definitions of composite adherence for the concurrent use of medications, including (a) the average of adherence, as measured by proportion of days covered (PDC), to each medication ("average"); (b) adherent only if $\geq 80 \%$ PDC, measured separately for each medication, on each concurrent medication ("all"); and (c) adherent if $\geq 80 \%$ PDC measured as proportion of days when at least 1 medication was available-i.e., a patient is adherent on a day if he or she possesses at least 1 medication on the day ("at least 1 "). ${ }^{7}$ In addition to the difference in perspective for each of these approaches, the measurement of adherence for multiple medications is further complicated depending on the definition of individual intervals, i.e., denominators of adherence measures. Specifically, the interval of individual medications can be based on the entire observation period or on a more limited prescription time period for each medication. However, because medications are to be continued, the prescription time period-based interval may not be important for most chronic disease medications except for situations when physician-recommended switches occur.

A single or composite estimate of adherence to multiple medications based on 1 of the these approaches or some variant of them has been used in several studies. Khanna et al. (2012) adopted the "at least 1" approach in the study of adherence to medications although the objective was not to examine multiple medication adherence. ${ }^{8} \mathrm{~A}$ study examining long-term adherence behavior among Medicaid patients averaged adherence rates of multiple medications for diabetes. ${ }^{9}$ Piette et al. (2007) employed a weighted average, analogous to the "average" measure, in the calculation of adherence to 2 intradisease medications for patients treated for multiple diseases-the weight was based on the adjustment (of days supply needed) made in the denominator of the estimator (i.e., medication possession ratio [MPR]), and the weighted average MPR was then dichotomized. ${ }^{10}$ Similarly, drug class adherence, measured by continuous measure of medication gaps, was combined into a single estimate for all medications for a chronic disease; the summary measure was computed by weighting the estimate for each class by the number of days from the first to last fill in the observation period and then the single estimate was dichotomized. ${ }^{11}$ Martin et al. (2009) presented a definition of PDC that the authors contended to have occurred because of therapeutic augmentation. ${ }^{12}$ The conservative variant of the definition is appropriate if it is believed that all medications are required as prescribed in order to elicit desired therapeutic effects. For quarters in which multiple medications were recommended, a patient was categorized as adherent on a day when he or she possessed all medications concurrently recommended and had

\section{TABLE 1 Composite Measurement Approaches to Intradisease Multiple Medication Adherence}

\begin{tabular}{|c|c|}
\hline Measure & Operational Definition \\
\hline Average $^{a}$ & {$\left[\frac{\text { Days with SU in Qtr i }}{\text { Days in Qtr } i}+\frac{\text { Days with TZD in Qtr } i}{\text { Days in Qtr } i}\right] / 2$} \\
\hline At least $1^{\mathrm{a}}$ & Adherent if $\left[\frac{\text { Days in Qtr } i \text { with } \geq 1 \text { medication available }}{\text { Days in Qtr i }}\right] \geq 80 \%$ \\
\hline Maximumb & {$\left[\frac{\text { Days in Qtr } i \text { with } \geq 1 \text { medication available }}{\text { Days in Qtr i }}\right]$} \\
\hline Both & Adherent if $\left[\frac{\text { Days in Qtr i with both medications available }}{\text { Days in Qtr i }}\right] \geq 80 \%$ \\
\hline Minimum $^{c}$ & {$\left[\frac{\text { Days in Qtr i with both medications available }}{\text { Days in Qtr } i}\right]$} \\
\hline Alla & $\begin{array}{l}\text { Adherent if }\left[\frac{\text { Days with SU in Qtr } i}{\text { Days in } 2 \operatorname{tr} i} \times 100 \%\right] \geq 80 \% \\
\text { and }\left[\frac{\text { Days with TZD in } 2 \operatorname{tr} i}{\text { Days in } 2 \operatorname{tr} i} \times 100 \%\right] \geq 80 \%\end{array}$ \\
\hline \multicolumn{2}{|c|}{$\begin{array}{l}\text { "Adapted from Choudhry NK, Shrank WH, Levin RL, et al. Measuring concurrent } \\
\text { adherence to multiple related medications. } \\
\text { bContinuous estimate of the "at least 1" approach. } \\
\text { "Continuous estimate of the "both" approach. } \\
\text { Qtr=quarter; SU = sulfonylureas; TZD = thiazolidinediones. }\end{array}$} \\
\hline
\end{tabular}

$80 \%$ or higher PDC as estimated by the method during the observation period (termed as "both"). Table 1 summarizes these concepts of composite measures of adherence to multiple medications.

It is not surprising that different measurement methods result in different adherence estimates, at least in some cases; however, it is important to estimate the extent of variability. A study in diabetes patients receiving multiple oral hypoglycemic agents reported that concurrent medication adherence estimates could range from 35\%-95\% depending on the composite measurement approaches followed. ${ }^{7}$ However, past research did not examine the adherence to individual medications in situations where multiple medications were prescribed for a disease and, more importantly, the effect of measurement algorithm selection on patient classification or overall adherence estimates. Because of the complexities of estimation of multiple medication adherence, patients taking 2 or more medications for a disease were excluded from analysis in many studies. ${ }^{10,13}$ Although measurement of adherence to multiple medications is of clinical significance, it has not been studied extensively. Currently, there is little consensus, and there exists no known standards or guidelines for measuring adherence to multiple medications, including those prescribed for chronic disease management. Thus, an examination of adherence and composite measures of adherence in situations where patients are prescribed multiple medications for a disease is important for understanding patient behavior and the impact of different composite measurement algorithms. 
The objectives of this study were to estimate (a) adherence to multiple medications prescribed for a disease; (b) composite adherence to multiple medications prescribed for a disease; and (c) the rate of differential classification as estimated by different algorithms.

\section{Methods}

\section{Study Design and Sample Description}

A longitudinal observational study design was employed using MarketScan Commercial Claims and Encounters data for 2002 and 2003. The data source represents medical and prescription claims of millions of private sector employees and their dependents. Enrollment, prescription claims, and inpatient and outpatient medical claims files were used. ${ }^{14}$ National Drug Code numbers from prescription claims records of selected patients were merged with their respective active ingredient (i.e., drug) names and strengths using the Multum Lexicon Drug Data Table. ${ }^{15}$

For this study, the identification of patients occurred from January 1, 2002, through September 30, 2002. Multiple medications are frequently prescribed for the treatment of diabetes. Thus, diabetes was considered an appropriate disease state for examining intradisease multiple medication adherence measurement. Patients who filled at least 1 prescription of each of 2 diabetes medications (i.e., sulfonylureas [SU] and thiazolidinediones [TZD]) that were available as separate pharmaceutical formulations were identified from prescription claims records. Such a filling pattern would provide an indication that patients were prescribed both medications. Furthermore, the earliest fill date (or later of 2 initial fills) indicating that patients were prescribed both medications served as the index date. Because of their complementary mechanisms of action, it was assumed that physicians were unlikely to replace 1 medication with the other even when the latest fill date for 1 and the earliest fill date for the other occur at a considerable temporal distance. Patients identified thus far were included in the study if they met the following inclusion criteria: (a) aged 18 years or older on the index date; (b) less than aged 65 years during the entire study period; (c) continuously enrolled in the database with pharmacy and medical benefits for at least 15 months (i.e., five 90-day quarters) starting from their respective index date; and (d) no record of filling other antidiabetic medications, including insulin, after the respective index date and during the study period. Patients were included if they filled a prescription for insulin or other antidiabetic medicines than SUs and TZDs only after the minimum 15-month period. In such cases, patients were not followed after they initiated such fills.

\section{Measurement of Adherence}

For this study, adherence was measured as PDC and estimated based on days' supply for each class of medications in each of the eight 90-day quarters of the observation period. ${ }^{6,7}$ The value of the days' supply was truncated in the event that the supply extended beyond the observation period. Any switches between different therapeutic agents (molecules) were not carried forward; however, equivalent agents (e.g., different brands of the same molecule) were carried forward. While the assumption in case of the former was that the physician modified the therapy regimen and the patient was not supposed to consume any leftover quantities of the medication that was discontinued, the assumption in the latter (e.g., between generic brands of the same therapeutic molecule) was that the patient would continue taking the medication from previous fills as part of the same treatment regimen. Patients may or may not have had a complete last quarter. For the last quarter, the number of available days of observation for a subject was entered in the respective denominator. Six composite measures as described in Table 1 were computed in each quarter. Six cumulative composite PDC estimates were calculated by proportion of days on medications out of all days until the end of each quarter or observation period. For categorical adherence measures, patients were considered adherent if $\mathrm{PDC} \geq 80 \%{ }^{6}$

Patients with prescription claims indicating duplicate fillings of medications were excluded from subsequent analysis. For example, some patients filled multiple medications or strengths of SUs or TZDs-fillings of multiple classes or strengths of SUs and/or TZDs somewhat regularly or switching from 1 strength to another within a medication. In such cases, patients were restricted to any molecule switch and/or strength switch within a molecule once only. That is, each type of (i.e., molecule and dosage strength) switch was constrained to once, if any, for every patient. This would ensure that if patients switched from 1 strength to another of the same medication or changed from 1 medication to a different one, they would not be filling 2 strengths or 2 classes of medications simultaneously. Thus, these patients would be on 2 diabetes medications, a SU and a TZD, at any point in time consistent with the objective of examining simultaneous medication adherence for 2 diabetes medications.

\section{Analysis Plan}

Descriptive statistics were calculated to describe the study population and examine the study objectives. The life-table method was used to compare survival functions (i.e., maintaining PDC $\geq 80 \%$ ) as estimated by the 3 different categorical composite cumulative measures ("at least 1," "both," "all"). The earliest (full) quarter in which composite cumulative PDC became $<80 \%$ was identified as time of discontinuation; thus, event times were grouped into intervals. Adjustment (Šidák correction) of $P$ values was made for pairwise comparisons of the survival functions generated by the 3 measures. Data were analyzed using SAS software, version 9.3 (SAS Institute, Inc., Cary, NC). 


\section{FIGURE 1 Selection of Patients}

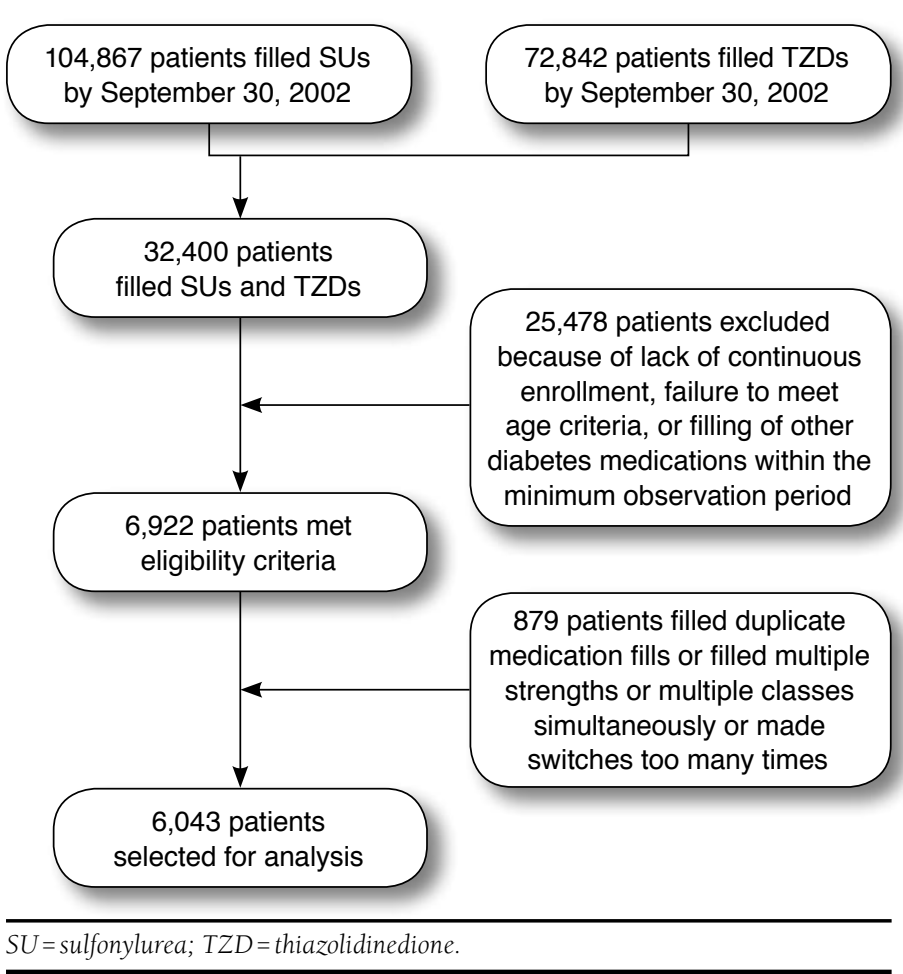

\section{Results}

Of all patients whose data were available in the MarketScan data utilized in this study, 32,400 enrollees had filled at least 1 prescription for an SU and a TZD by September 30, 2002. After the application of study inclusion criteria, 6,922 patients were eligible for subsequent analysis (Figure 1). Finally, after including the filling pattern restriction requirements, 6,043 patients were available for analysis and constituted the final study sample. The demographic characteristics of the patients are outlined in Table 2. Approximately half of the sample was male (55.5\%), and the average age was nearly 54 years. Patients in preferred provider organizations and comprehensive health plans comprised approximately $70 \%$ of the sample, and only $11.3 \%$ had changed their plan types over the enrollment period. Approximately $63 \%$ and $15 \%$ of patients filled prescriptions of SUs and TZDs only from community pharmacies and mail order pharmacies, respectively.

\section{Intradisease Multiple Medication Adherence: Individual Adherence Estimates}

Overall, as was the aim of the first objective, patients demonstrated a mean PDC estimate of approximately $74 \%$ for both SUs and TZDs and maintained general consistency in adherence between the 2 medications over time (Table 3). With

\begin{tabular}{|c|c|c|}
\hline $\begin{array}{ll}\text { TABLE } 2 \text { Demog } \\
\text { of the } 5\end{array}$ & tharac & \\
\hline Variable & $\mathrm{N}$ & $\begin{array}{l}\text { Mean (SD)/ } \\
\text { Frequency (\%) }\end{array}$ \\
\hline Age & 6,043 & $53.9(6.7)$ \\
\hline Gender (male) & 3,356 & 55.5 \\
\hline \multicolumn{3}{|l|}{ Geographic region $^{\mathrm{a}}$} \\
\hline Northeast & 523 & 8.7 \\
\hline North Central & 2,442 & 40.4 \\
\hline South & 2,580 & 42.7 \\
\hline West & 488 & 8.1 \\
\hline \multicolumn{3}{|l|}{ Plan type ${ }^{b}$} \\
\hline Comprehensive & 1,862 & 30.8 \\
\hline EPO & 161 & 2.7 \\
\hline $\mathrm{HMO}$ & 588 & 9.7 \\
\hline POS & 883 & 14.6 \\
\hline $\mathrm{PPO}$ & 2,789 & 46.2 \\
\hline POS with capitation & 462 & 7.6 \\
\hline \multicolumn{3}{|l|}{ Number of plan type } \\
\hline 1 type & 5,359 & 88.7 \\
\hline 2 or more & 684 & 11.3 \\
\hline \multicolumn{3}{|l|}{ Pharmacy patronage $^{c}$} \\
\hline Community pharmacy only & 3,776 & 62.5 \\
\hline Mail order only & 893 & 14.8 \\
\hline Either type & 1,135 & 18.8 \\
\hline Number of days of observation & 6,043 & $636.2(79)$ \\
\hline \multicolumn{3}{|c|}{$\begin{array}{l}\text { aAs per enrollment summary in } 2002 \text { and } 10 \text { patients from unknown regions. } \\
\text { bSome patients reported having multiple types of plan in a year, so the percentages } \\
\text { add to more than } 100 \% \text {. }\end{array}$} \\
\hline \multicolumn{3}{|c|}{$\begin{array}{l}\text { cPercentages do not add to } 100 \% \text { and represent filling of sulfonylureas and thiazoli- } \\
\text { dinediones in cases that identified pharmacy type. }\end{array}$} \\
\hline \multicolumn{3}{|c|}{$\begin{array}{l}E P O=\text { exclusive provider organization; } H M O=\text { health maintenance organization; } \\
P O S=\text { point of service; } P P O=\text { preferred provider organization; } S D=\text { standard deviation. }\end{array}$} \\
\hline
\end{tabular}

regard to population-level estimates, SU adherence levels measured at each quarter closely followed TZD adherence levels for the respective quarters such that the maximum difference in rates between SUs and TZDs was less than 2\%. All patients had 90-day observation periods at least until the fifth quarter; however, the number of available days of observation in their last quarters varied among patients. As expected, adherence rates appeared to slightly decline over time until quarter 6 . However, the adherence rates increased slightly for the last 2 quarters, although the number of patients available for observation continued to decrease.

\section{Intradisease Multiple Medication Adherence: Composite Adherence Estimates}

Table 4 provides quarter specific and cumulative PDC estimates as calculated by the continuous measure-based approaches outlined in the second objective. Population estimates of PDC varied depending on measurement time. For example, population PDC estimates for quarter 5 were $82.8 \%$, 59.2\%, and $71.0 \%$ based on the maximum (i.e., availability of any 
TABLE 3 PDC Estimates for SUs and TZDs

\begin{tabular}{|c|c|c|c|c|c|}
\hline \multirow[b]{2}{*}{ Quarter } & \multirow[b]{2}{*}{$\mathrm{N}$} & \multicolumn{2}{|c|}{ SUs } & \multicolumn{2}{|c|}{ TZDs } \\
\hline & & Mean & SD & Mean & SD \\
\hline 1 & 6,043 & 84.2 & 23.9 & 85.6 & 22.5 \\
\hline 2 & 6,043 & 75.0 & 32.8 & 75.8 & 33.1 \\
\hline 3 & 6,043 & 73.8 & 34.0 & 74.0 & 34.8 \\
\hline 4 & 6,043 & 72.9 & 35.2 & 72.5 & 36.4 \\
\hline 5 & 6,043 & 71.7 & 36.1 & 70.3 & 37.9 \\
\hline 6 & $6,043^{a}$ & 69.8 & 37.8 & 69.0 & 39.4 \\
\hline 7 & $4,979^{a}$ & 70.0 & 38.2 & 69.4 & 39.6 \\
\hline 8 & $3,752^{\mathrm{a}}$ & 72.2 & 38.5 & 70.8 & 40.0 \\
\hline Weighted average ${ }^{b}$ & \multicolumn{3}{|c|}{73.9} & \multicolumn{2}{|c|}{73.7} \\
\hline \multicolumn{6}{|c|}{$\begin{array}{l}\text { aNumbers of days of observation in these quarters are not } 90 \text { days for all patients } \\
b \text { Weighted by } N \text {. } \\
P D C=\text { proportion of days covered; } S D=\text { standard deviation; } S U=\text { sulfonylurea; } \\
T Z D=\text { thiazolidinedione. }\end{array}$} \\
\hline
\end{tabular}

medications on a day), minimum (i.e., availability of all medications on a day), and average algorithm-based approaches, respectively. Similarly, cumulative PDC estimates differed; cumulative PDC estimates for the eighth quarter with subjectspecific variable observation periods were $87.4 \%, 67.3 \%$, and $77.4 \%$ as calculated by the maximum, minimum, and average PDC measures, respectively. Variations of quarterly adherence estimates (see standard deviations) based on the minimum measurement were generally more than those of the other 2 approaches.

Single measures of multiple medication adherence were computed on a dichotomous scale with a cut-point of $80 \%$ to classify patients as adherent. There was considerable variability in estimates measured by the "at least 1" (i.e., $80 \%$ days on at least 1 medication); "both" (i.e., $80 \%$ days on both medications); and "all" (i.e., $80 \%$ days on each medication measured separately) approaches. The percentages of patients who were classified as adherent ranged from $88.2 \%$ at quarter 1 to $78.0 \%$ at quarter 8 when measured by the "at least 1" approach. Similarly, estimates of adherent subjects dropped from $61.2 \%$ to $51.0 \%$ for "both," and $62.9 \%$ to $51.9 \%$ for "all" from quarter 1 to quarter 8 (see Table 5).

\section{Discrepancy in Classification}

Using dichotomous measures of adherence, a patient can be classified as adherent or nonadherent. Thus, a person can be classified as adherent by only 1 composite dichotomous measure, 2 measures, or all 3 measures. To examine the discrepancy in classification provided by different dichotomy-based approaches, additional analyses were conducted on quarterspecific PDC and included patients who were rated adherent by at least 1 of the measurement approaches (see Table 6). More than $30 \%$ of patients were differentially classified at all

\begin{tabular}{|c|c|c|c|c|c|c|}
\hline \multirow[b]{2}{*}{ proach } & \multirow[b]{2}{*}{ Quarter } & \multirow[b]{2}{*}{$\mathrm{N}$} & \multicolumn{2}{|c|}{ Quarter-Specific } & \multicolumn{2}{|c|}{ Cumulative } \\
\hline & & & Mean & SD & Mean & SD \\
\hline \multirow{8}{*}{ ximum } & 1 & 6,043 & 93.3 & 14.9 & 93.3 & 14.9 \\
\hline & 2 & 6,043 & 86.7 & 24.9 & 90.0 & 18.1 \\
\hline & 3 & 6,043 & 85.5 & 26.8 & 88.5 & 19.5 \\
\hline & 4 & 6,043 & 84.4 & 28.5 & 87.5 & 20.6 \\
\hline & 5 & 6,043 & 82.8 & 30.3 & 86.5 & 21.5 \\
\hline & 6 & 6,043 & 81.4 & 32.2 & 85.8 & 22.1 \\
\hline & 7 & 4,979 & 81.6 & 32.4 & 86.5 & 21.3 \\
\hline & 8 & 3,752 & 83.1 & 32.2 & 87.4 & 20.5 \\
\hline \multirow{8}{*}{ nimum } & 1 & 6,043 & 76.5 & 28.3 & 76.5 & 28.3 \\
\hline & 2 & 6,043 & 64.1 & 37.3 & 70.3 & 29.9 \\
\hline & 3 & 6,043 & 62.3 & 38.3 & 67.7 & 30.6 \\
\hline & 4 & 6,043 & 61.0 & 39.5 & 66.0 & 31.2 \\
\hline & 5 & 6,043 & 59.2 & 40.2 & 64.6 & 31.5 \\
\hline & 6 & 6,043 & 57.4 & 41.5 & 63.5 & 31.8 \\
\hline & 7 & 4,979 & 57.7 & 42.0 & 64.9 & 31.0 \\
\hline & 8 & 3,752 & 59.9 & 42.8 & 67.3 & 30.0 \\
\hline \multirow{8}{*}{ erage } & 1 & 6,043 & 84.9 & 19.3 & 84.9 & 19.3 \\
\hline & 2 & 6,043 & 75.4 & 28.0 & 80.2 & 21.7 \\
\hline & 3 & 6,043 & 73.9 & 29.4 & 78.1 & 22.8 \\
\hline & 4 & 6,043 & 72.7 & 30.7 & 76.7 & 23.7 \\
\hline & 5 & 6,043 & 71.0 & 31.9 & 75.6 & 24.3 \\
\hline & 6 & 6,043 & 69.4 & 33.4 & 74.6 & 24.7 \\
\hline & 7 & 4,979 & 69.7 & 33.6 & 75.7 & 24.0 \\
\hline & 8 & 3,752 & 71.5 & 33.7 & 77.4 & 23.3 \\
\hline
\end{tabular}

$P D C=$ proportion of days covered; $S D=$ standard deviation .

times. In other words, about one-third of the study sample was rated as adherent based on 1 measurement approach but nonadherent based on another approach. Rates of such differential classification remained high (34.6\%-37.7\%) in later quarters despite decreasing sample size.

A life-table analysis (Figure 2) was performed to compare how different categorical composite measures classified population persistence estimates based on whether subjects demonstrated PDC at a rate of $80 \%$ or more cumulatively. In this analysis, patients were observed until the respective last full quarter (i.e., quarter with an observation period of 90 days) and followed until they became nonadherent (i.e., cumulative PDC $<80 \%$ ) for the first time or censored at the end of the last full quarter. The "at least 1" approach identified more patients as persistent and showed a much slower decline than did the "all" or "both" approaches, both of which closely followed each other. In addition, the gap widened over time. A log-rank test with Šidák correction for multiple comparisons revealed that all pairwise comparisons of the 3 approaches were statistically significant $(P<0.05)$, indicating differences in survival function of one from that of another. 
Adherence to Multiple Medications Prescribed for a Chronic Disease: A Methodological Investigation

TABLE 5 Estimates of Adherent Subjects Classified by Different Dichotomous Composite Measures

\begin{tabular}{|c|c|c|c|c|c|c|c|c|c|c|}
\hline \multirow[b]{2}{*}{ Approach } & \multirow[b]{2}{*}{ Quarter } & \multirow[b]{2}{*}{$\mathrm{N}$} & \multicolumn{4}{|c|}{ Quarter-Specific } & \multicolumn{4}{|c|}{ Cumulative } \\
\hline & & & \multicolumn{2}{|c|}{$\begin{array}{c}\text { Nonadherent } \\
\text { n (\%) }\end{array}$} & \multicolumn{2}{|c|}{$\begin{array}{l}\text { Adherent }^{\mathrm{a}} \\
\text { n (\%) }\end{array}$} & \multicolumn{2}{|c|}{$\begin{array}{c}\text { Nonadherent } \\
\text { n (\%) }\end{array}$} & \multicolumn{2}{|c|}{$\begin{array}{l}\text { Adherent }^{\mathrm{a}} \\
\text { n (\%) }\end{array}$} \\
\hline \multirow{8}{*}{ At least 1} & 1 & 6,043 & 716 & (11.9) & 5,327 & $(88.2)$ & 716 & (11.9) & 5,327 & $(88.2)$ \\
\hline & 2 & 6,043 & 1,199 & $(19.8)$ & 4,844 & $(80.2)$ & 967 & $(16.0)$ & 5,076 & $(84.0)$ \\
\hline & 3 & 6,043 & 1,293 & $(21.4)$ & 4,750 & $(78.6)$ & 1,156 & (19.1) & 4,887 & $(80.9)$ \\
\hline & 4 & 6,043 & 1,356 & $(22.4)$ & 4,687 & $(77.6)$ & 1,209 & $(20.0)$ & 4,834 & $(80.0)$ \\
\hline & 5 & 6,043 & 1,465 & $(24.2)$ & 4,578 & $(75.8)$ & 1,275 & $(21.1)$ & 4,768 & $(78.9)$ \\
\hline & 6 & 6,043 & 1,543 & $(25.5)$ & 4,500 & $(74.5)$ & 1,393 & $(23.1)$ & 4,650 & $(77.0)$ \\
\hline & 7 & 4,979 & 1,233 & $(24.8)$ & 3,746 & $(75.2)$ & 1,091 & $(21.9)$ & 3,888 & $(78.1)$ \\
\hline & 8 & 3,752 & 826 & $(22.0)$ & 2,926 & $(78.0)$ & 777 & $(20.7)$ & 2,975 & $(79.3)$ \\
\hline \multirow{8}{*}{ Both } & 1 & 6,043 & 2,346 & $(38.8)$ & 3,697 & $(61.2)$ & 2,346 & (38.8) & 3,697 & $(61.2)$ \\
\hline & 2 & 6,043 & 2,977 & (49.3) & 3,066 & $(50.7)$ & 2,868 & $(47.5)$ & 3,175 & $(42.5)$ \\
\hline & 3 & 6,043 & 3,108 & $(51.4)$ & 2,935 & $(48.6)$ & 3,132 & $(51.8)$ & 2,911 & $(48.2)$ \\
\hline & 4 & 6,043 & 3,125 & $(51.7)$ & 2,918 & $(48.3)$ & 3,253 & $(53.8)$ & 2,790 & $(46.2)$ \\
\hline & 5 & 6,043 & 3,200 & $(53.0)$ & 2,843 & $(47.1)$ & 3,345 & $(55.4)$ & 2,698 & $(44.7)$ \\
\hline & 6 & 6,043 & 3,240 & (53.6) & 2,803 & $(46.4)$ & 3,423 & (56.6) & 2,620 & (43.4) \\
\hline & 7 & 4,979 & 2,599 & $(52.2)$ & 2,380 & (47.8) & 2,766 & (55.6) & 2,213 & $(44.5)$ \\
\hline & 8 & 3,752 & 1,837 & $(49.0)$ & 1,915 & $(51.0)$ & 1,999 & (53.3) & 1,753 & $(46.7)$ \\
\hline \multirow{8}{*}{ All } & 1 & 6,043 & 2,241 & (37.1) & 3,802 & (62.9) & 2,241 & (37.1) & 3,802 & $(62.9)$ \\
\hline & 2 & 6,043 & 2,868 & $(47.5)$ & 3,175 & $(52.5)$ & 2,681 & (44.4) & 3,362 & (55.6) \\
\hline & 3 & 6,043 & 3,004 & $(49.7)$ & 3,039 & $(50.3)$ & 2,952 & $(48.9)$ & 3,091 & $(51.2)$ \\
\hline & 4 & 6,043 & 3,029 & $(50.1)$ & 3,014 & $(49.9)$ & 3,037 & $(50.3)$ & 3,006 & (49.8) \\
\hline & 5 & 6,043 & 3,112 & $(51.5)$ & 2,931 & $(48.5)$ & 3,085 & (51.1) & 2,958 & $(49.0)$ \\
\hline & 6 & 6,043 & 3,161 & $(52.3)$ & 2,882 & $(47.7)$ & 3,201 & $(53.0)$ & 2,842 & $(47.0)$ \\
\hline & 7 & 4,979 & 2,549 & $(51.2)$ & 2,430 & $(48.8)$ & 2,579 & $(51.8)$ & 2,400 & $(48.2)$ \\
\hline & 8 & 3,752 & 1,805 & $(48.1)$ & 1,947 & $(51.9)$ & 1,847 & (49.2) & 1,905 & $(50.8)$ \\
\hline
\end{tabular}

\section{Discussion}

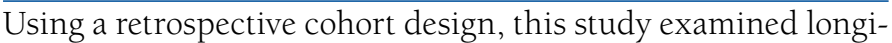
tudinally adherence to multiple medications prescribed for the treatment of a disease. As medication adherence is a time-varying behavior, patient observation over time is believed to have generated knowledge that will be useful to improve chronic disease management. Results of this study demonstrated that patient adherence to each medication, although not poor, may not be considered optimal by conventional standards. In general, the population adherence estimates of individual medications mirrored each other closely. It is plausible that there is a strong correlation between simultaneous adherence to more than 1 medication at the individual patient level. This conclusion implies that adherence to 1 medication is likely to be associated with adherence to another medication. However, such a conclusion requires empirical evidence in order to avoid the ecological fallacy. Interestingly, adherence estimates of each medication in this cohort remained relatively stable and even improved slightly in the last 2 quarters of the observation period. It is possible that this cohort, apart from having insurance coverage and access to health care services, demonstrates stable medication consumption behavior over time. Future studies should investigate these factors further.
Measurement of adherence to concurrent multiple medications is a complex issue. Generally, a diverse set of approaches that are followed add further complexities to results from said analyses and in the interpretation of adherence estimates. The means of (single) continuous PDC estimates of multiple medication adherence varied widely depending on the type of measurement used. Both average and minimum-based approaches yielded suboptimal (per $\geq 80 \%$ criterion) adherence rates. These differences were present when PDCs were calculated at each quarter or estimated cumulatively. That the extent of variability in adherence estimates was greatly affected by the algorithm employed (e.g., range and standard deviations of estimates by the "minimum" approach or requiring patients to possess both medications was larger than those of others) implies that such variability would continue to grow in some cases (e.g., populations with overall adherence rates lower than the population used in the study or instances where a patient is followed longer than 8 quarters). Indeed, such variability should be already at play in some cases and recognized for the sake of ensuring uniform care strategies across patients, times, or populations.

Results from single dichotomous measures of multiple medication adherence ( $\geq 80 \%$ PDC) were consistent with find- 
TABLE 6

Measure of Discrepancy

\begin{tabular}{l|c|c}
\hline \multirow{2}{*}{ Quarter } & \multicolumn{2}{|c}{ Quarter-Specific } \\
\cline { 2 - 3 } & $\mathrm{N}^{\mathrm{a}}$ & $\%$ \\
\hline 1 & 1,630 & 30.6 \\
\hline 2 & 1,778 & 36.7 \\
\hline 3 & 1,815 & 38.2 \\
\hline 4 & 1,769 & 37.7 \\
\hline 5 & 1,735 & 37.9 \\
\hline 6 & 1,697 & 37.7 \\
\hline 7 & 1,366 & 36.5 \\
\hline 8 & 1,011 & 34.6 \\
\hline a Number of patients considered adherent by 1 or 2 dichotomous composite mea- \\
sures but not by all 3 approaches.
\end{tabular}

ings from continuous measure-based composite estimates, demonstrating considerable variability in number of adherent subjects based on the approach used. The "all" estimates were slightly smaller but were closely followed by the estimates measured by the "both" approach, which provided the lowest estimates. It is important to note that cumulative dichotomous composite measures are conceptually measures of persistence. Thus, estimates produced by them are similar to persistent estimates if we were to apply respective composite algorithms on a persistent measure. Although a small difference was identified between "all" and "both," all 3 approaches were significantly different from one another. Fortunately, per Pharmacy Quality Alliance (PQA) measure specifications, the "at least 1" algorithm is advocated for computing Medicare Part D adherence measures when patients are found to be taking more than 1 intradisease medication; at a minimum, it imposes a degree of consistency in the methodology of adherence rate calculation in the Medicare population across health plans. In other words, PQA Medicare Part D measures will yield methodologically consistent adherence estimates that are comparable with regard to different variables. Thus, the contribution made by Part D measures toward health plan rating can be compared and uniformly interpreted. Hypothetically, though, if there are differences in the rate of concurrent medication use and/or different composite measurement algorithms are applied in other cases, it has the potential to yield different estimates of adherence or patients who were persistent. Thus, choice of measurement approach does matter for the purposes of interpretation of adherence estimates. In particular, utilization of adherence research for disease management decision making in clinical practice settings will be challenging when there is a lack of a consistently defined measurement approach.

Importantly, not every composite dichotomous measure will consistently classify patients as adherent or nonadherent unless patients demonstrate high adherence rates (typically $80 \%$ or more). Consistent with previous research, this study
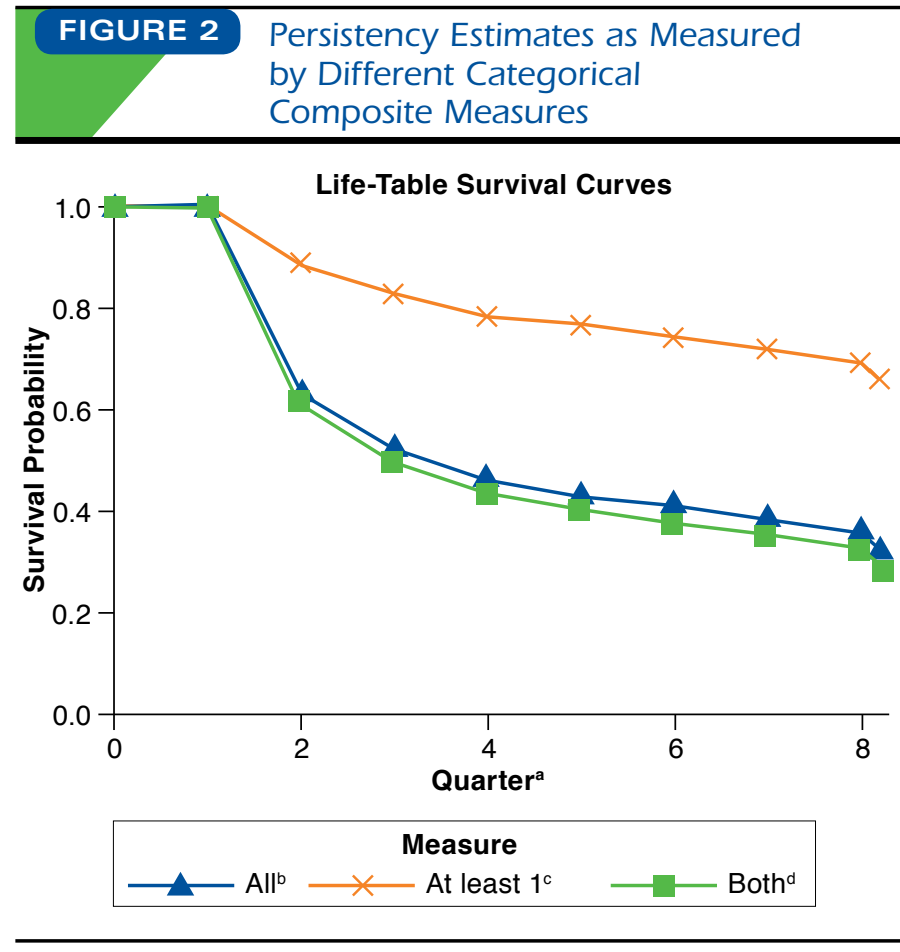

aPatients were considered persistent until cumulative adherence estimate fell below $80 \%$ in a quarter.

bAll: $80 \%$ of days covered by each medication measured separately.

cAt least 1: $80 \%$ of days covered by at least 1 medication.

dBoth: $80 \%$ of days covered by both medications.

has found variability in adherence estimates contingent upon measurement methods. ${ }^{7}$ This study quantified and compared the variability that may result because of applying different composite algorithms. It was found that the extent of discrepancy in classification of patients who were rated adherent at least by 1 , but not by all, of the approaches could be as high as $38.2 \%$. Such discrepancy will become more apparent if overall population adherence rates become poor or when a longer observation period is considered. More importantly, the extent of disparate qualitative interpretations may pose decision-making issues, such as whether and when to intervene, for practitioners or disease-management professionals. For example, if a health care provider believes that a patient must adhere to all medications as prescribed, he or she may consider only the "both" definition, while another provider may believe a patient should follow at least 1 of the regimens in order to be considered adherent. In addition, there are other numerous factors (e.g., desired health outcomes, health status) that may influence such decision making. In reality, practitioners will receive adherence estimates, in cases provided as part of quality monitoring, without much knowledge about estimation method, which should ideally be founded upon clinical 
rationale. Evidence-based guidelines on composite adherence measurement are required for appropriate and consistent patient care for chronic disease management.

The current study extends the literature on adherence measurement for chronic disease medications in several important ways. First, to our knowledge, only 1 previous study has examined the impact of applying different definitions of composite measures on adherence estimates. ${ }^{7}$ The results of the current study corroborate those findings, which empirically demonstrated variability in a cohort of diabetes patients. Unlike the previous study, this study employed a restricted operational definition of concurrent adherence in which patients were allowed to be on 2 intradisease medications with additional restrictions on switches. Thus, the variability in adherence due to measurement methods is believed to have been more precisely measured and driven more by patient nonadherence behavior than other factors, such as potential overcounting occurring from the medications that were discontinued. In addition, such restrictions would improve generalizability in prescribing situations where 2 concurrent medications are advised. As the advent of newer classes of medications enhances the likelihood of using multiple medications, these study findings will be directly applicable to future treatment modalities. Second, the present study offers a new method to compute a composite measure of concurrent adherence, i.e., the "both" algorithm. Although this definition is the most restrictive among the others compared, it is certainly less conservative if medication adherence is defined as adhering to a regimen exactly as prescribed, including taking the exact dosage and taking medication the correct number of times a day. Third, the current study estimated the simultaneous adherence behaviors for each individual medication. In the treatment of chronic diseases that often offer the opportunity to use multiple medications that are only available as separate dosage forms, the results of this study offer guidance on patent behaviors. Finally, this study explicitly demonstrates the potential of adherence misclassification depending on algorithms of concurrent adherence behavior measurement. This study also quantifies the extent of inconsistent or differential classifications and indicates the ways in which measurement algorithms result in statistically different adherence estimates. If intervention decisions are mired by measurement fallacies, quality of care at the individual level or population level and long-term public health will be deleteriously affected.

\section{Limitations}

While the study results highlight key points on adherence measurement, it is important to consider the findings in light of methodological limitations. First, limitations embedded in characteristics of administrative claims data (e.g., coding qual- ity and completeness event recording) are applicable to this study. Second, many patients had less than a 90-day observation period at their respective last quarter. As the number of available days of observation in the last quarter was used as the denominator, it may have inflated adherence rates in later quarters. Third, it was not possible to identify patients who were initiating SU and TZD therapy. Because newly initiated patients may show different adherence behaviors from those with longer treatment history, this may have affected adherence estimates. However, a past study that used the same data source indicates that a large number of patients were prescribed the dual therapy even before the start of the observation period of this study. ${ }^{16}$ Fourth, the data used in the study were from prescription claim records from 2002-2003. If adherence behavior has changed substantially in recent years, the results may differ from what would be found with a newer dataset. However, as medication adherence is as much of an issue now as it has been for several decades, it was not anticipated that the data affected the results related to adherence behaviors. Moreover, the dataset used is believed to have no effect on the part of the results related to the effect of composite measurement algorithms. In fact, the data facilitated the operationalization of multiple medications that were available as separate dosage formulations. Finally, the study sample was composed of patients enrolled in a commercial claims database of private sector employees. Therefore, further research is required before the study results can be generalized to other payer systems or uninsured populations.

\section{Conclusions}

Adherence to multiple medications prescribed for a disease (i.e., intradisease multiple medication adherence) is a complex health behavior issue. Patients in this study showed levels of adherence to individual medications for a chronic disease that do not satisfy the conventional definition of adherence; thus, they cannot be universally considered as adherent. Given that a number of operationalizations of composite adherence exist, the interpretation of composite adherence estimates and findings across studies become difficult. Although health care quality initiatives — whether it is pay for performance or quality improvement programs-encourage monitoring of adherence, the disparate use of composite adherence measures creates challenges for researchers regarding the appropriateness of measures to be used in adherence studies and for physicians and pharmacists when implementing clinical and patient care decision making. Although useful, composite measures that are neither adopted uniformly nor based on clinical rationale have the potential to disproportionately affect quality of care and public health. 


\section{Authors}

RAMSANKAR BASAK, PhD, is a Research and Analytics Professional in a privately held company, Annapolis Junction, Maryland. DAVID J. MCCAFFREY, III, RPh, PhD, is Assistant Dean and Professor, St. John Fisher College School of Pharmacy, Rochester, New York. JOHN P. BENTLEY, PhD, is Professor, Department of Pharmacy Administration; DONNA WEST-STRUM, $R P h, P h D$, is Chair and Associate Professor, Department of Pharmacy Administration; and BENJAMIN F. BANAHAN, III, PhD, is Professor and Director, Center for Pharmaceutical Marketing and Management, The University of Mississippi, Oxford, Mississippi. SARAHMONA M. PRZYBYLA, PhD, is a Postdoctoral Research Associate, The University at Buffalo Research Institute on Addictions, Buffalo, New York.

AUTHOR CORRESPONDENCE: Ramsankar Basak, PhD, P.O. Box 473, Annapolis Junction, MD 20701. Tel.: 662.801.6484; E-mail:basakrams@gmail.com.

\section{DISCLOSURES}

Basak received funding for this project from the University of Mississippi Graduate Student Council. Truven Health Analytics provided data access support through the dissertation support program. The authors declare no potential conflicts of interest regarding this research.

Study concept and design was primarily contributed by Basak, McCaffrey, and Banahan, with assistance from Bentley, Przybyla, and West-Strum. Data were collected by Basak and McCaffrey and interpreted by Bentley and Basak, with input from McCaffrey, Przybyla, West-Strum, and Banahan. The manuscript was written primarily by Basak, with assistance from the rest of the authors, and revised by Basak, Przybyla, and West-Strum, with assistance from McCaffrey, Bentley, and Banahan.

\section{ACKNOWLEDGMENTS}

The authors wish to thank Truven Health Analytics, formerly Thomson Reuters Healthcare, Inc., for providing data through the dissertation support program at no cost.

\section{REFERENCES}

1. Leeper SC. Aggressive hypertension management in patients of advancing and advanced age. South Med J. 2005;98(8):805-08.
2. Mustone Alexander L. Desirable therapeutic characteristics of an optimal antihypertensive agent. Drugs. 2006;66(9):1239-52

3. Sokol MC, McGuigan KA, Verbrugge RR, Epstein RS. Impact of medication adherence on hospitalization risk and healthcare cost. Med Care. 2005;43(6):521-30.

4. Osterberg L, Blaschke T. Adherence to medication. N Engl J Med. 2005;353(5):487-97.

5. Benner JS, Glynn RJ, Mogun H, Neumann PJ, Weinstein MC, Avorn J. Long-term persistence in use of statin therapy in elderly patients. JAMA. 2002;288(4):455-61.

6. Fairman K, Motheral B. Evaluating medication adherence: which measure is right for your program? J Manag Care Pharm. 2000;6(6):499-504. Available at: http://www.amcp.org/data/jmcp/ce_v6_499-506.pdf.

7. Choudhry NK, Shrank WH, Levin RL, et al. Measuring concurrent adherence to multiple related medications. Am J Manag Care. 2009;15(7):457-64.

8. Khanna R, Pace PF, Mahabaleshwarkar R, Basak RS, Datar M, Banahan BF. Medication adherence for chronic diseases among recipients enrolled in a state Medicaid program. Popul Health Manag. 2012;15(5):253-60.

9. Yu AP, Yu YF, Nichol MB. Estimating the effect of medication adherence on health outcomes among patients with type 2 diabetes-an application of marginal structural models. Value Health. 2010;13(8):1038-45.

10. Piette JD, Heisler M, Ganoczy D, McCarthy JF, Valenstein M. Differential medication adherence among patients with schizophrenia and comorbid diabetes and hypertension. Psychiatr Serv. 2007;58(2):207-12.

11. Schmittdiel JA, Uratsu CS, Karter AJ, et al. Why don't diabetes patients achieve recommended risk factor targets? Poor adherence versus lack of treatment intensification. J Gen Intern Med. 2008;23(5):588-94.

12. Martin BC, Wiley-Exley EK, Richards S, Domino ME, Carey TS, Sleath BL. Contrasting measures of adherence with simple drug use, medication switching, and therapeutic duplication. Ann Pharmacother. 2009;43(1):36-44.

13. Karve S, Cleves MA, Helm M, Hudson TJ, West DS, Martin BC. An empirical basis for standardizing adherence measures derived from administrative claims data among diabetic patients. Med Care. 2008;46(11):1125-33.

14. MarketScan Research Databases. Truven Health Analytics (formerly Thomson Reuters Healthcare) Inc., Ann Arbor, MI. Available at: https://marketscan.truvenhealth.com/marketscanportal/. Accessed June 21, 2014.

15. Cerner Multum. Lexicon Plus. 2011. Denver, CO.

16. Cohen FJ, Neslusan CA, Conklin JE, Song X. Recent antihyperglycemic prescribing trends for US privately insured patients with type 2 diabetes. Diabetes Care. 2003;26(6):1847-51 